УДК 004.9

\title{
Нейросетевой кластерный анализ в среде NeuroXL Clusterizer
}

Осипов Геннадий Сергеевич

Сахалинский государственный университет

д.т.н., заведующиий кафедрой Информатики

Вамакидзе Нателла Семеновна

Сахалинский государственный университет

дочент кафедры Информатики

\section{Аннотация}

Сформулированы методологические аспекты использования самоорганизующихся сетей Кохонена для решения задачи разделения множества объектов на кластеры. Предложено в качестве инструментария для проведения исследования использовать надстройку над Excel NeuroXL Clusterizer компании OLSOFT LLC. Рассмотрен практический пример кластеризации совокупности стран по набору показателей.

Ключевые слова: кластерный анализ, нейронная сеть.

\section{Neural network cluster analysis in NeuroXL Clusterizer environment}

Osipov Gennadij Sergeevich

Sakhalin State University

Doctor of technical Sciences, Head of the Department of Computer Science

Vashakidze Natella Semenovna

Sakhalin State University.

Associate Professor of Department of Computer Science

\section{Abstract}

Methodological aspects of the use of self-organizing Kohonen networks for solving the problem of dividing a set of objects into clusters are formulated. It was proposed to use the add-in for Excel NeuroXL Clusterizer of Olsoft LLC as a tool for conducting the research. A practical example of clustering of a set of countries by a set of indicators is considered.

Keywords Cluster analysis, neural network

\section{Введение}

Современные достижения в области искусственного интеллекта во многом базируются на использовании искусственных нейронных сетей. Особое положение здесь занимают самоорганизующиеся нейронные сети, или сети (карты, решетки) Кохонена, которые способны менять свою топологию подстраиваясь под структуру поступающих на них исходных 
данных. По сути, это сети, которые «обучаются без учителя», т.е. являются самоорганизующимися.

Одной из наиболее важных и трудоемких задач интеллектуальной обработки информации является проблема разделения исследуемых объектов на группы, классы или кластеры (в многопараметрической постановке) для их последующей классификации и дальнейших исследований. Разделение на классы позволяет в перспективе идентифицировать новый объект и применить для его изучения отработанные методы исследований.

Таким образом данная работа имеет целью отработать основные методологические аспекты нейросетевого подхода к решению задач кластерного анализа. Исследования являются продолжением работ, представленных в [1,2].

\section{Постановка задачи}

Имеется множество систем (объектов, ситуаций), характеризуемых набором параметров. Требуется разбить исходное множество на непересекающиеся подмножества, которые будут содержать в известном смысле близкие друг-другу системы.

Количество подмножеств (кластеров) и степень «компактности» каждого из них могут задаваться пользователем или выбираться автоматически в соответствии с используемым алгоритмом кластеризации.

\section{Инструментарий}

Задачи кластеризации и классификации достаточно часто возникают и решаются на практике стандартными «жесткими» алгоритмами, которые не допускают возможности адаптации и своей самоорганизации.

Наибольших успехов отрасль искусственного интеллекта, связанная с проблемой синергетической самоорганизации, достигла благодаря использованию самоорганизующихся сетей (карт, решеток) Кохоненеа, которые относятся к классу искусственных нейронных сетей, функционирующих «без учителя» и способных самостоятельно за счет изменения собственной пространственной топологии эффективно решать задачи разделения исходного множества данных на классы (кластеры, сгустки).

В качестве инструментария для проведения разработки выбрана надстройка над Excel NeuroXL Clusterizer [3], которая позволяет пользователю работать в знакомой программной среде и не требует дополнительных знаний из теории нейронных сетей.

\section{Практическая реализация}

Решим задачу, сформулированную в [3]. Множество стран, характеризуется унифицированным набором характеристик (параметров - A, B, C, D, E, F), представленных на рисунке 1. 


\begin{tabular}{|l|c|c|c|c|c|c|}
\hline \multicolumn{1}{|c|}{ Страна } & A & B & C & D & E & F \\
\hline Алжир & 3,9 & 31193917 & 1,74 & 2381740 & 0 & 5550 \\
\hline Аргентина & -3 & 36955182 & 1,16 & 2736690 & 30200 & 17000 \\
\hline Австралия & 4,3 & 19169083 & 1,02 & 7617930 & 68920 & 21070 \\
\hline Бразилия & 0,8 & 172860370 & 0,94 & 8456510 & 55455 & 28000 \\
\hline Канада & 3,6 & 31281092 & 1,02 & 9220970 & 755170 & 7100 \\
\hline Китай & 7 & 1261832482 & 0,9 & 9326410 & 270550 & 498720 \\
\hline Индия & 5,5 & 1014003817 & 1,58 & 2973190 & 314400 & 480000 \\
\hline Индонезия & 0 & 22484210 & 1,63 & 1826440 & 93000 & 45970 \\
\hline Иран & 1 & 65619636 & 0,83 & 1636000 & 12000 & 94000 \\
\hline Казахстан & 1,7 & 16733227 & $-0,05$ & 2669800 & 47500 & 22000 \\
\hline Ливия & 2 & 5115450 & 2,42 & 1759540 & 0 & 4700 \\
\hline Мексика & 3,7 & 100349766 & 1,53 & 1923040 & 49510 & 61000 \\
\hline Монголия & 3,5 & 2650952 & 1,54 & 1565000 & 0 & 800 \\
\hline Нигерия & 2 & 10075511 & 2,75 & 1266700 & 300 & 660 \\
\hline Перу & 2,4 & 27012899 & 1,75 & 1280000 & 5220 & 12800 \\
\hline Россия & 3,2 & 146001176 & $-0,38$ & 16995800 & 79400 & 40000 \\
\hline Саудовская Аравия & 1,6 & 22023508 & 3,28 & 1960582 & 0 & 4350 \\
\hline Судан & 3 & 35079814 & 2,84 & 2376000 & 129810 & 19460 \\
\hline СшА & 4,1 & 275562673 & 0,91 & 9158960 & 470131 & 207000 \\
\hline
\end{tabular}

Рис.1 Структура исходных данных в $M S$ Excel

На рисунке 2 представлено заполненное диалоговое окно используемой надстройки, в котором указан диапазон исходных и выходных данных, а также априори определено количество кластеров, на которые необходимо разбить исходной множество стран.

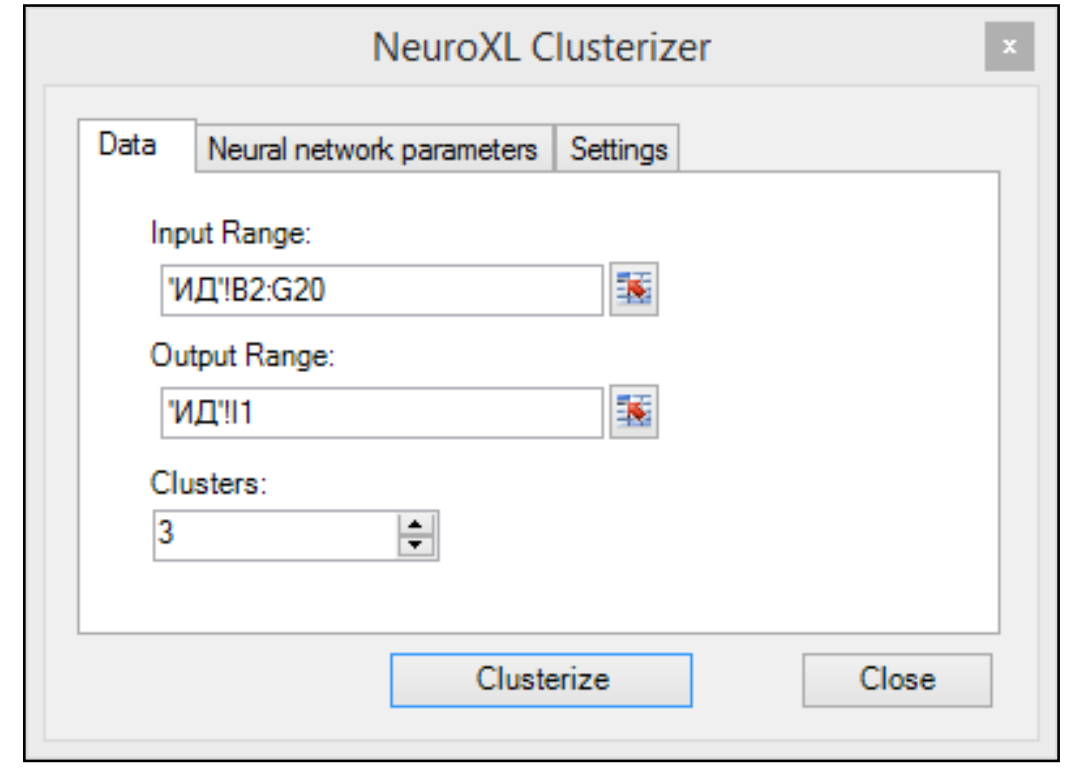

Рис. 2 Заполненная закладка по исходным данным

Закладки по настройке параметров нейронной сети можно (рис. 3) оставить без изменений (согласившись с «рекомендациями» программного средства по умолчанию) 


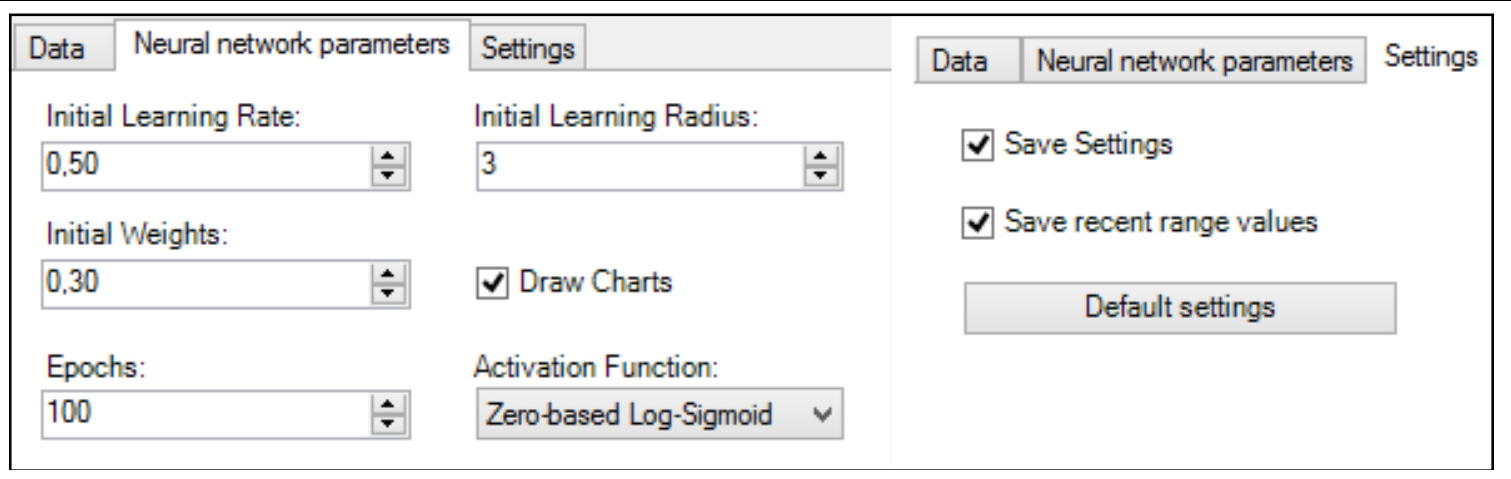

Рис. 3 Параметры настройки

После выполнения кластеризации множество стран автоматически отобразится в Excel с учетом их принадлежности к тому или иному кластеру (рис. 4), которые нумеруются и различаются цветом.

\begin{tabular}{|c|c|c|c|c|c|c|c|}
\hline Страна & A & B & C & D & $E$ & $\mathbf{F}$ & Кластеры \\
\hline Алжир & 3,9 & 31193917 & 1,74 & 2381740 & 0 & 5550 & 1 \\
\hline Аргентина & -3 & 36955182 & 1,16 & 2736690 & 30200 & 17000 & 1 \\
\hline Австралия & 4,3 & 19169083 & 1,02 & 7617930 & 68920 & 21070 & 1 \\
\hline Иран & 1 & 65619636 & 0,83 & 1636000 & 12000 & 94000 & 1 \\
\hline Казахстан & 1,7 & 16733227 & $-0,05$ & 2669800 & 47500 & 22000 & 1 \\
\hline Мексика & 3,7 & 100349766 & 1,53 & 1923040 & 49510 & 61000 & 1 \\
\hline Монголия & 3,5 & 2650952 & 1,54 & 1565000 & 0 & 800 & 1 \\
\hline Нигерия & 2 & 10075511 & 2,75 & 1266700 & 300 & 660 & 1 \\
\hline Перу & 2,4 & 27012899 & 1,75 & 1280000 & 5220 & 12800 & 1 \\
\hline Россия & 3,2 & 146001176 & $-0,38$ & 16995800 & 79400 & 40000 & 1 \\
\hline Судан & 3 & 35079814 & 2,84 & 2376000 & 129810 & 19460 & 1 \\
\hline США & 4,1 & 275562673 & 0,91 & 9158960 & 470131 & 207000 & 2 \\
\hline Бразилия & 0,8 & 172860370 & 0,94 & 8456510 & 55455 & 28000 & 2 \\
\hline Канада & 3,6 & 31281092 & 1,02 & 9220970 & 755170 & 7100 & 2 \\
\hline Ливия & 2 & 5115450 & 2,42 & 1759540 & 0 & 4700 & 2 \\
\hline Саудовская & 1,6 & 22023508 & 3,28 & 1960582 & 0 & 4350 & 3 \\
\hline Китай & 7 & 1261832482 & 0,9 & 9326410 & 270550 & 498720 & 3 \\
\hline Индия & 5,5 & 1014003817 & 1,58 & 2973190 & 314400 & 480000 & 3 \\
\hline Индонезия & 0 & 22484210 & 1,63 & 1826440 & 93000 & 45970 & 3 \\
\hline
\end{tabular}

Рис. 4 Преобразование исходных данных

Программа автоматически формирует и размещает на рабочем листе Excel информацию о весах и профилях кластеров (рис. 5 и 6). 


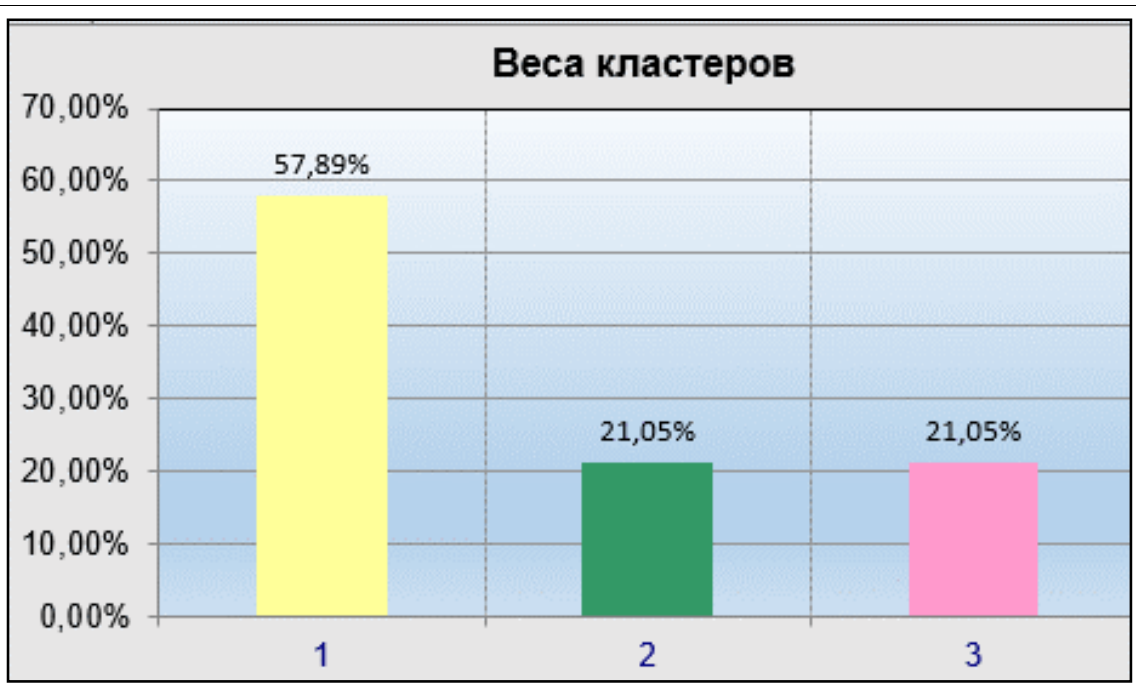

Рис.5 Процент количества объектов в кластерах

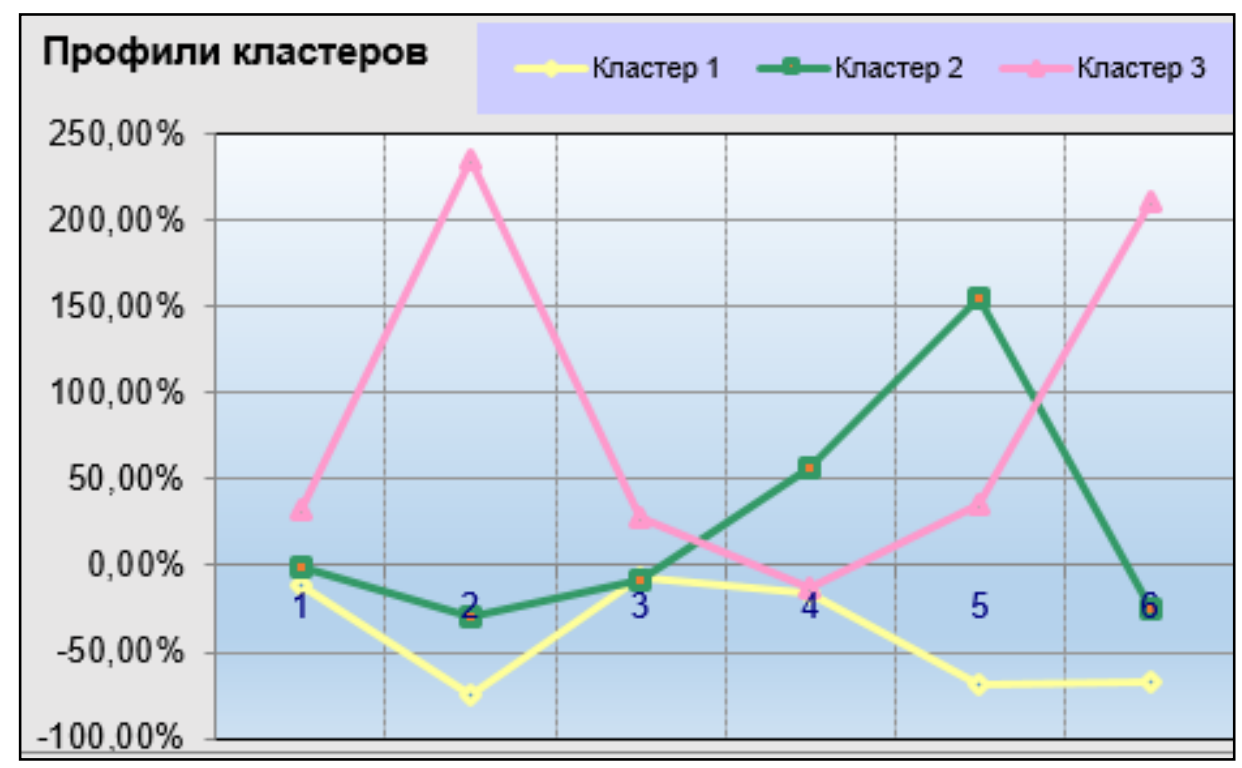

Рис. 6 Параметры кластеров

Кроме того, дополнительно выводится информация о минимальном, максимальном и среднем значении параметров в кластерах.

\section{Выводы}

Проведенное исследование позволяет сделать следующие выводы:

1. Проблема классификации и кластеризации объектов достаточно эффективно решается с помощью самоорганизующихся искусственных нейронных сетей.

2. Применение NeuroXL Clusterizer позволяет упростить решение задачи за счет того, что работа выполняется непосредственно в среде $M S$ Excel и, кроме того, от пользователя не требуются профессиональные знания по используемым нейронным сетям.

\section{Библиографический список}

1. Technical analysis software for Microsoft Excel. URL: 
http://www.analyzerxl.com/ (дата обращения: 13.08.2017).

2. Осипов Г.С., Вашакидзе Н.С., Филиппова Г.В. Основы прогнозирования финансовых временных рядов на базе NeuroXL Predictor // Постулат. 2017. № 7. C. 26.

3. NeuroXL Clusterizer. URL: http://neuroxl.com/products/excel-cluster-analysissoftware/neuroxl-clusterizer.htm (дата обращения: 13.08.2017). 